\title{
Etude des phénomènes pré- et post-zygotiques au sein du genre Phaseolus L. : cas de $P$. coccineus $\mathbf{L}$. et $P$. vulgaris $\mathbf{L}$.
}

\author{
Pamphile NGUEMA NDOUTOUMOU
}

\author{
Université des Sciences et Techniques de Masuku, Institut National Supérieur d'Agronomie et de \\ Biotechnologies (INSAB), BP : 99, Poto-Poto Franceville Gabon. \\ E-mail: pamphilen@hotmail.com; Tél.: (+241) 07711001
}

\section{RESUME}

Les graines constituent une part importante de l'alimentation humaine et animale dans le monde. L'embryogenèse végétale est, à ce titre, un important phénomène situé à l'amont de leur production. Du point de vue génétique, l'embryogenèse est également une étape importante de la conservation de la biodiversité et de l'augmentation de la variabilité existante. L'observation de l'embryogenèse indique qu'après la double fécondation, le zygote passe par les étapes de division cellulaire marquée par la multiplication et l'expansion des cellules. L'étape finale de ce processus est la déshydratation de la graine. Durant les différents stades embryogéniques, il est établi une corrélation entre la croissance et le développement embryonnaire. Cette évolution se traduit, d'une part, par la différenciation entre le suspenseur et l'embryon proprement dit, et d'autre part, par la spécialisation des tissus initiés. L'embryogenèse est similaire pour les deux espèces considérées dans ce travail. Les décalages sont relevés dans le délai de développement de l'embryon.

La connaissance des mécanismes qui régissent l'embryogenèse est donc nécessaire pour expliquer et contourner les barrières d'incompatibilité post-zygotiques observées lors des croisements interspécifiques au sein du genre Phaseolus L.

(C) 2009 International Formulae Group. All rights reserved.

Mots-clés : embryon végétal, Phaseolus, pollinisation, hybridations, division cellulaire.

\section{INTRODUCTION}

L'embryogenèse végétale précède la germination. Elle est essentielle pour la survie des espèces et fondamentale pour la vie du futur individu, car elle détermine sa formation et définit ses potentialités.

Son importance est également capitale pour l'agriculture et l'économie. En effet, elle mène à la formation des graines qui représentent la part principale de l'alimentation dans le monde.

L'embryogenèse a été largement étudiée et elle s'insère dans le cycle de vie des végétaux supérieurs, alternant une phase sporophytique diploïde et une phase gamétophytique haploïde.
À la suite de la fécondation, l'embryogenèse consiste à la formation de l'embryon en vue de l'établissement du plan de la future plante. Elle commence par une division du zygote en deux cellules selon une polarité base-apex prédéfinie (Tamborindeguy, 2004). Le déroulement de cette étape est sous le contrôle de nombreux mécanismes se rapportant soit à la génétique (Lopes et Larkins, 1993), soit à la physiologie (Massardo et al., 2000; Yeung et al., 2001) ou à d'autres facteurs extrinsèques à la plante.
D'une
manière
générale,

l'embryogenèse est constituée de trois étapes majeures chez les plantes à fleurs (Yeung et Meinke, 1993; Sornsathapornkul et Owens, 1999; Gallois, 2001; Yeung et al., 2001; Grini 
et al., 2002; Sallandrouze et al., 2002; Nomizu et al., 2004).

L'embryogenèse précoce est l'étape de morphogenèse qui aboutit à la formation de deux axes dont l'un traduit la mise en place des différents tissus et l'autre correspond à l'édification des organes conditionnant les aptitudes des futurs individus à survivre grâce à la possibilité de capter plus facilement la lumière (organes aériens) et à mieux coloniser le sol (système racinaire), à la recherche de nutriments. La maturation intervient plus tard et l'embryon emmagasine des réserves pour assurer la germination et se préparer à la dormance.

\section{MATERIEL ET METHODES}

\section{Matériel végétal, conditions de culture et pollinisations}

Les génotypes utilisés sont NI16 et NI1108 de P. coccineus, NI637 et X484 de $P$. vulgaris. Ils sont respectivement d'origine rwandaise, mexicaine, brésilienne et colombienne. Ils sont identifiés par leur numéro d'introduction (NI) ou leur numéro d'attente d'introduction (X) au Jardin Botanique National de Belgique. Les conditions et la conduite de la culture sont identiques à celles décrites par Nguema et al. (2007).

Les génotypes de $P$. vulgaris (NI637 et $\mathrm{X} 707$ ) sont autogames, tandis que la pollinisation est réalisée manuellement chez les génotypes de l'espèce allogame $P$. coccineus.

\section{Viabilité du pollen in vitro et réceptivité du stigmate}

Les fleurs sont collectées à l'anthèse pour le test de viabilité du pollen in vitro. Ensuite, les anthères sont prélevés et mis dans les Eppendorf contenant $1 \mathrm{ml}$ de milieu liquide de germination du pollen $(20 \%$ saccharose et $0,001 \%$ de $\left.\mathrm{H}_{3} \mathrm{BO}_{3}\right)$. Les Eppendorf sont secoués doucement pour libérer le pollen. Ce mélange est versé sur un milieu solide de germination (contenant $0,5 \%$ d'agar), puis mis dans le luminincube pendant 24 heures, à une luminosité voisine de 40 $\mu \mathrm{E} / \mathrm{m}^{2}$.s et une température de $21 \pm 1^{\circ} \mathrm{C}$.

Pour tester la réceptivité du stigmate, les fleurs autofécondées sont récoltées deux à quatre jours après la pollinisation, puis trempées dans de l'éthanol à $70 \%$ pendant 24 heures. Les pistils sont lavés durant 15 minutes à l'eau courante, et rincés pendant une heure dans une solution de $\mathrm{NaOH} 4 \mathrm{~N}$, ensuite, maintenues dans du bleu d'aniline durant toute la nuit.

Les ovules sont ensuite isolés des ovaires et placés sur une lame avec le style et le stigmate. Dans une goutte d'eau, ils sont écrasés délicatement entre lame et lamelle, puis examinés au microscope à fluorescence en utilisant une excitation de lumière bleue $(455 \mathrm{~nm})$. Le pollen et les tubes polliniques sont identifiés par la fluorescence spécifique de la callose sur les parois cellulaires. Les observations se font au microscope de marque Nikon (Eclipse, E800), aux grossissements 10 et 20 .

\section{Protocole histologique, paramètres et analyse statistique}

Le protocole histologique est celui décrit par Nguema et al. (2009).

Dans ce travail, le terme "embryon" se réfère au suspenseur et à la partie organogénétique diploïde de l'embryon dénommé "embryon proprement dit". Le suspenseur est arbitrairement divisé en deux zones pour faciliter la description. La base du suspenseur est élargie et rattachée au tissu maternel. Sa partie supérieure, plus étroite constitue le corps du suspenseur. Les mesures portent sur des coupes longitudinales axiales et médianes d'embryons.

Les cellules du suspenseur sont comptées. Sa longueur et sa surface sont mesurées à partir du micropyle jusqu'à la jonction entre le suspenseur et l'embryon proprement dit. La longueur de l'embryon est prise suivant l'axe longitudinal, de la base du suspenseur jusqu'à l'extrémité de l'embryon. Sa largeur est prise perpendiculairement à l'axe précédent.

Les stades successifs de développement de l'embryon peuvent être identifiés. Il s'agit notamment des stades globulaire, cordiforme et cotylédonaire (Brady et Combs, 1998).

La description des principales structures embryonnaires se rapporte notamment au stade de développement de l'embryon, la présence de cellules de l'albumen, l'intégrité de l'endothélium, l'aspect présenté par la base du suspenseur et le suspenseur lui-même. 
Pour chaque structure embryonnaire observée chez le génotype X484, cinq mesures sont prises sur trois ovules provenant de différents ovaires d'une même plante. Les valeurs moyennes des paramètres considérés sont traitées statistiquement par une analyse de variance, à l'aide du logiciel MINITAB 14, à un seuil de signification de $95 \%$.

\section{RESULTATS ET DISCUSSION}

Les principaux acteurs de la fécondation et les évènements pré-zygotiques

La formation du gamétophyte mâle est initiée par les cellules mères de microspores, ou microsporocytes. Ces cellules se développent à l'intérieur des anthères. Les microsporocytes subissent la méiose, produisant chacun quatre microspores haploïdes. Chaque microspore se divise une première fois en une cellule végétative et une cellule générative (Figures 1 et 2 ). Cette structure bicellulaire est le microgamétophyte immature ou grain de pollen. Dans les premiers instants de la pollinisation, la cellule générative se divise et donne deux gamètes mâles. Ils sont entraînés par le tube pollinique jusqu'à l'extrémité micropylaire de l'ovule. Le grain de pollen germé, avec son tube et ses deux gamètes, représente le gamétophyte mâle adulte. Il est l'acteur mâle de la double fécondation.

Le sporophyte produit des spores haploïdes par divisions méiotiques comme l'ont également décrit de nombreux auteurs tels que Whittier et Braggins (2000), Derksen et al. (2002), Teixeira et al. (2004), Ledesma et Sugiyama (2005), et Shi et Stösser (2005).

Parallèlement au développement du pollen, la formation du gamétophyte femelle se déroule dans l'ovule. Des gamètes haploïdes sont produits par réduction méiotique. L'ovaire contient un ou plusieurs ovules. Au début, l'ovule en développement est formé d'un pied appelé funicule et d'un nucelle. Il s'y ajoute rapidement une ou deux enveloppes qui sont les téguments, ménageant une petite ouverture à la base appelée le micropyle (Figures 3 et 4).

Dans l'ovule, l'oosphère est entourée de deux synergides et, à l'opposé se trouvent les antipodes. Les deux autres noyaux sont ceux de la cellule centrale. Le sac embryonnaire est entouré par les téguments interne et externe, qui constitueront la coque protectrice de la graine. Tous ces tissus participent au développement de l'embryon et de l'albumen.

Avant la fécondation, le sac embryonnaire est quasi vide, mais au terme de l'embryogenèse, il sera complété par les cotylédons qui accumulent des réserves amylacées.

À l'intérieur de l'ovule, un seul mégasporocyte se développe et subit finalement la méiose, donnant naissance à quatre mégaspores, dont trois dégénèrent. La quatrième poursuit son développement et à maturité, elle devient une structure à sept cellules et huit noyaux. Le sac embryonnaire se retrouve ainsi au sein de l'ovule qui est une structure sporophytique multicellulaire, conformément aux observations de Anderson et Hill (2002), Stauffer et al. (2002), Berg (2003), Farjon et Ortiz (2003), Pullman et Buchanan (2003), et Soltis et Soltis (2004).

Le développement embryonnaire chez Phaseolus L.

Embryogenèse de $P$. vulgaris

Dès l'anthèse, le pollen germe sur la surface stigmatique, puis émet un tube pollinique qui se déplace dans le tissu stylaire (Figure 2) et ovarien avant de pénétrer l'ovule par le micropyle. Les éléments du sac embryonnaire sont prêts à recevoir le gamétophyte mâle (Figure 4).

Plusieurs phénomènes physiques (pression osmotique, fusion cellulaire, croissance des cellules en volume et en masse, etc.) et physiologiques (pont calcique, activité hormonale, mobilisation des enzymes, etc.) interagissent au moment de la pénétration du micropyle par le tube pollinique. Lorsque ce dernier atteint le sac embryonnaire, il pénètre l'une des synergides en précipitant sa destruction. Puis la double fécondation aboutit à la formation de deux cellules: le zygote (diploïde) et l'albumen primaire (triploïde). La première division du zygote est asymétrique.

Le zygote ou proembryon est composé d'un petit nombre de cellules. Les premières divisions de cet embryon globoïde ou préglobulaire (Figure 5) aboutissent à la formation d'un embryon globulaire qui va atteindre le stade cordiforme (Figure 6) au bout de quelques jours. Par la suite, les cotylédons s'initient (Figure 8). C'est le stade cotylédonaire (Figure 9), suivi de la 


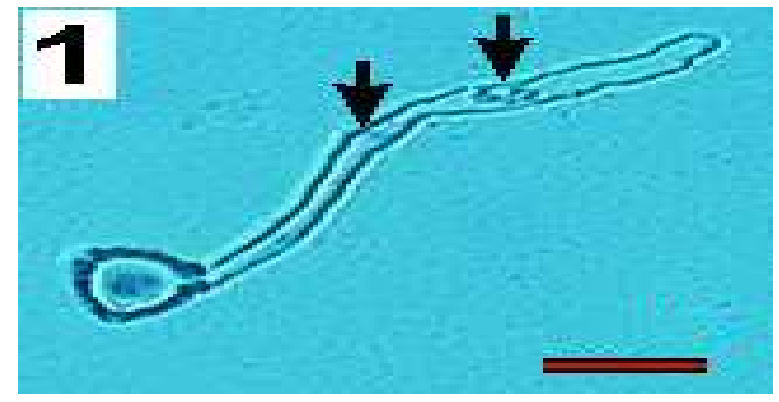

Figure 1 : Germination in vitro du pollen chez $P$. vulgaris. Le pollen a germé et le tube pollinique a atteint une taille d'environ $250 \mu \mathrm{m}, 24$ heures après sa culture in vitro. Les deux noyaux, l'un végétatif et l'autre génératif, sont visibles vers la partie distale du grain de pollen $(\downarrow)(-=50 \mu \mathrm{m})$.

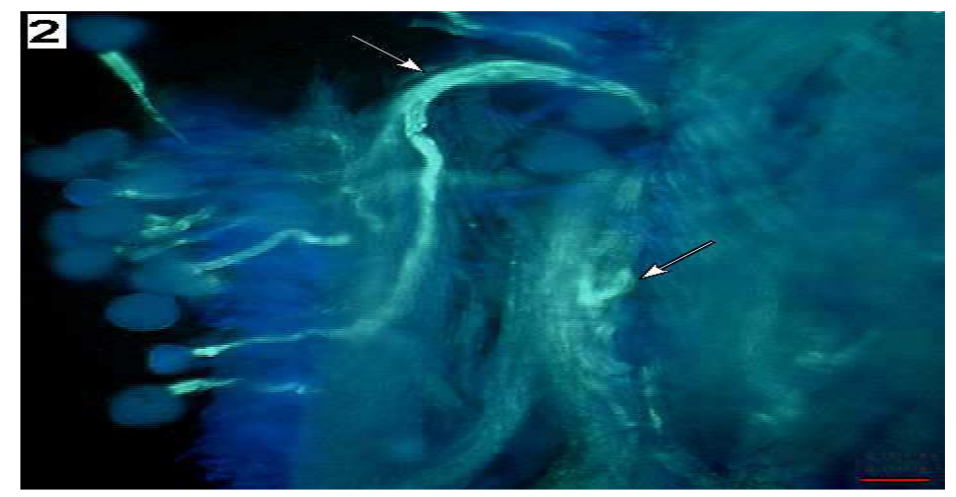

Figure 2 : Observation in situ de la croissance du tube pollinique chez $P$. vulgaris. Le pollen a germé sur la surface stigmatique. L'observation à la fluorescence permet de voir des tubes polliniques pénétrant le style. La callose contenue dans les tubes polliniques $(\downarrow)$ favorise le suivi de leur parcours $(-=50 \mu \mathrm{m})$.

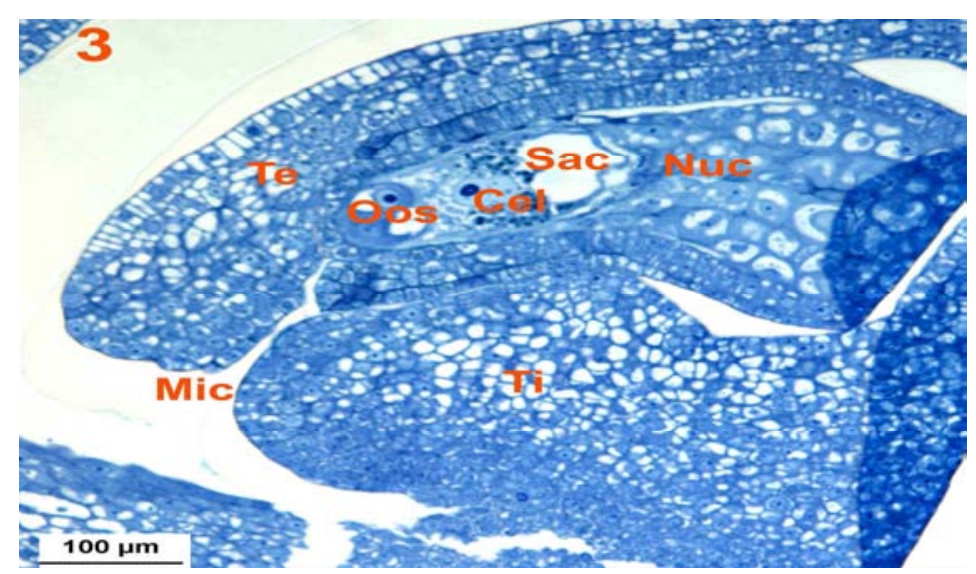

Figure 3 : Coupe longitudinale médiane d'un ovule de $P$. vulgaris. Un jour avant l'anthèse, les cellules des antipodes ont dégénéré à proximité du nucelle (nuc), du côté chalazien. Le noyau de la cellule mère $(\mathrm{Oo})$ est visible et proche du micropyle (mic). Au milieu du sac embryonnaire (sac), on voit l'un des noyaux de la cellule centrale $(\mathrm{cc})$. Le nucelle occupe une grande partie à l'intérieur de l'ovule. Les téguments interne (Ti) et externe (Te), puis l'endothélium (end) sont visibles. 


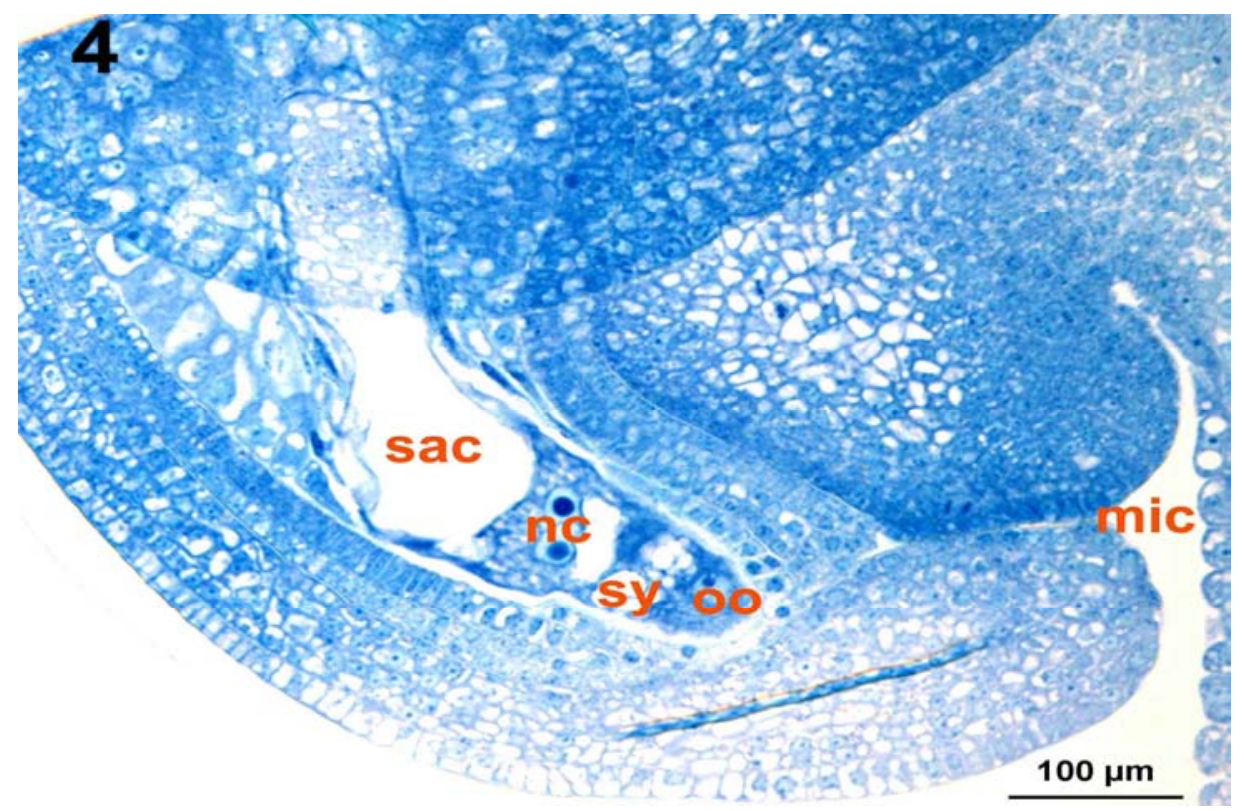

Figure 4 : Coupe longitudinale médiane d'un ovule de $P$. vulgaris. À l'anthèse, les principales cellules destinées à la double fécondation sont matures dans le sac embryonnaire (sac). Les noyaux de la cellule centrale (nc), l'oosphère (Oo) et l'une des synergides (syn) sont prêts pour la fécondation.

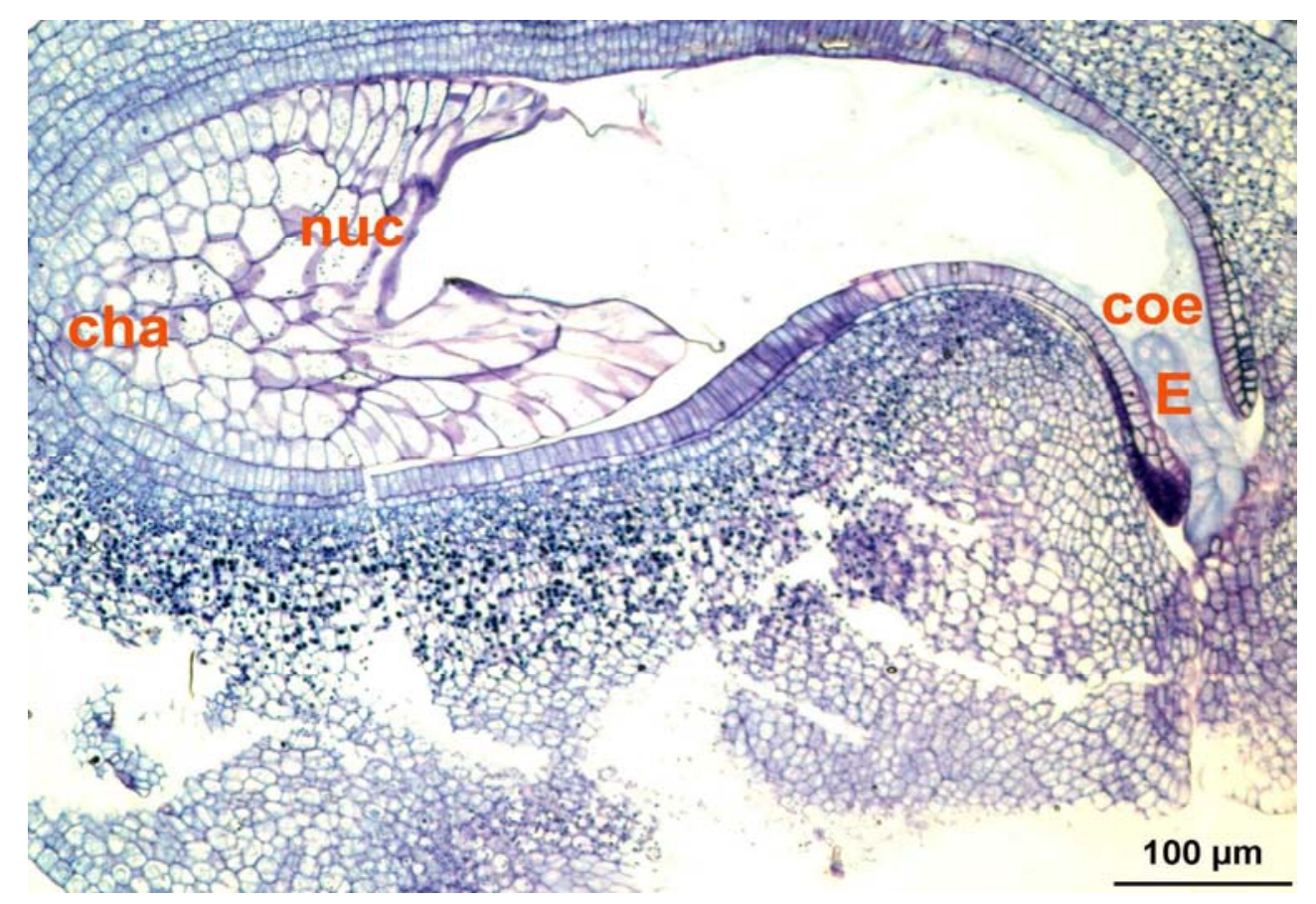

Figure 5 : Coupe longitudinale médiane d'un ovule de $P$. vulgaris. L'embryon (E) est globoïde ou pré-globulaire. Il est recouvert d'albumen coenocytique (coe). Le côté micropylaire se trouve sous la partie basale de l'embryon. La forme en $\mathbf{U}$ du nucelle (nuc) est observée vers la chalaze (cha). 


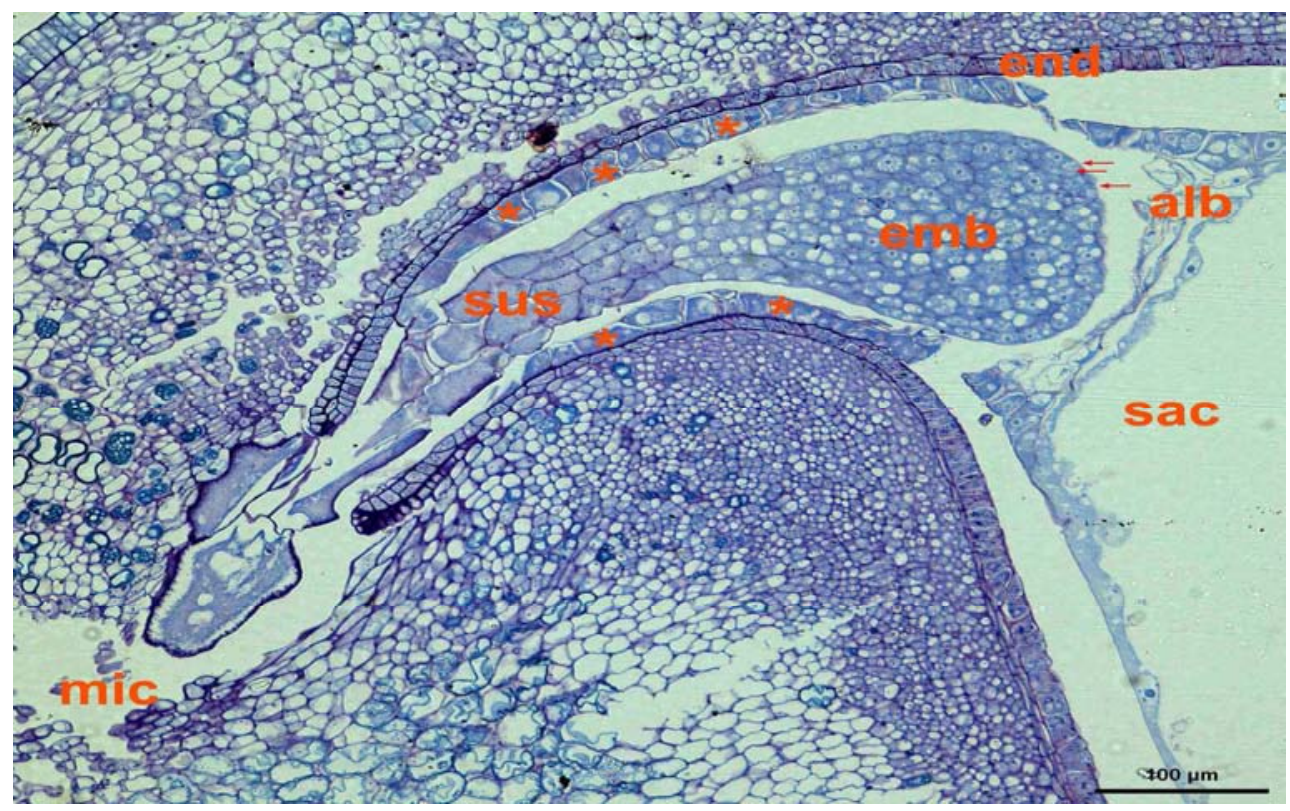

Figure 6: Coupe longitudinale médiane dans un ovule de $P$. vulgaris montrant un embryon cordiforme âgé. Un filet d'albumen (alb) sépare le sac embryonnaire (sac) de l'embryon proprement dit (emb). La base du suspenseur (sus) est faite de cellules allongées vers le micropyle (mic). Les cellules de transfert $(*)$ sont logées entre l'endothélium (end) et l'embryon. Le protoderme $(\downarrow)$ délimite la partie apicale de l'embryon.

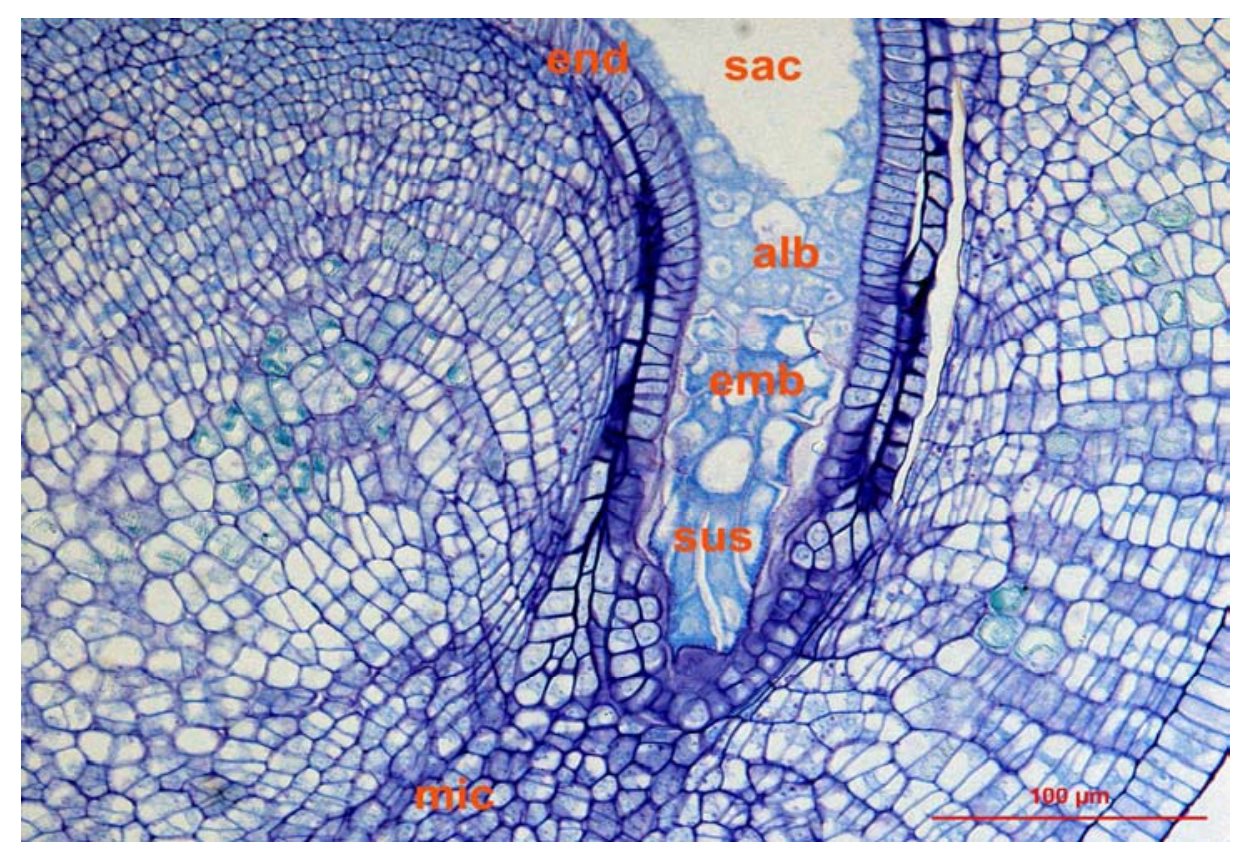

Figure 7 : Coupe longitudinale médiane dans un ovule de P. coccineus montrant un embryon globulaire (emb). Une structure lâche faite de cellules albuminées (alb) entoure l'embryon. L'albumen cellulaire est visible jusqu'au suspenseur (sus), vers le micropyle (mic). Les divisions de l'albumen se poursuivent dans le sac embryonnaire (sac) et le long de la paroi endothéliale (end). 


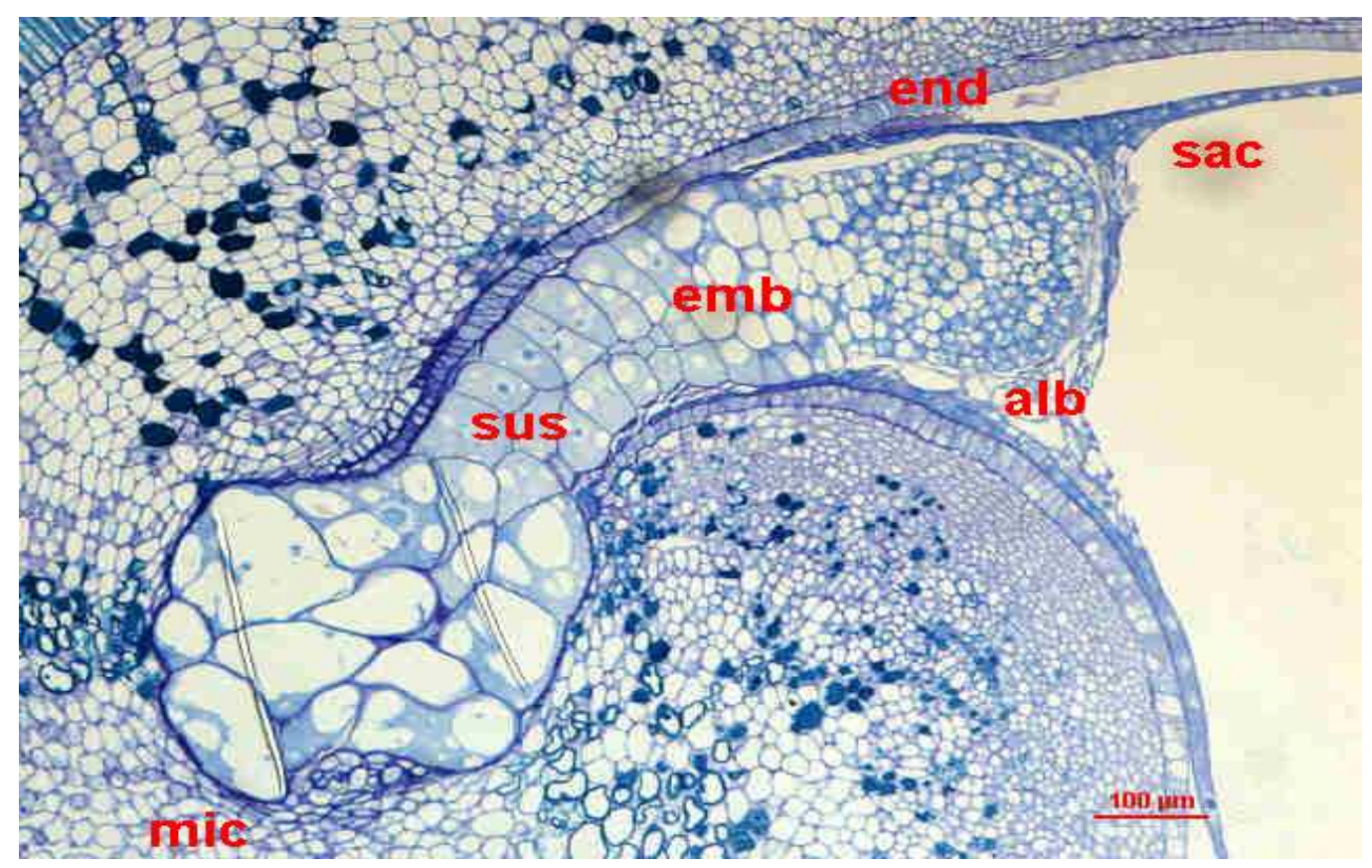

Figure 8 : Coupe longitudinale médiane dans un ovule de $P$. coccineus. L'embryon (emb) a atteint le stade cordiforme tardif. Le suspenseur (sus) montre des cellules imposantes à sa base, du côté micropylaire (mic). L'embryon est séparé de l'endothélium (end) par une fine cloison. Des cellules d'albumen (alb) sont visibles dans le sac embryonnaire (sac).

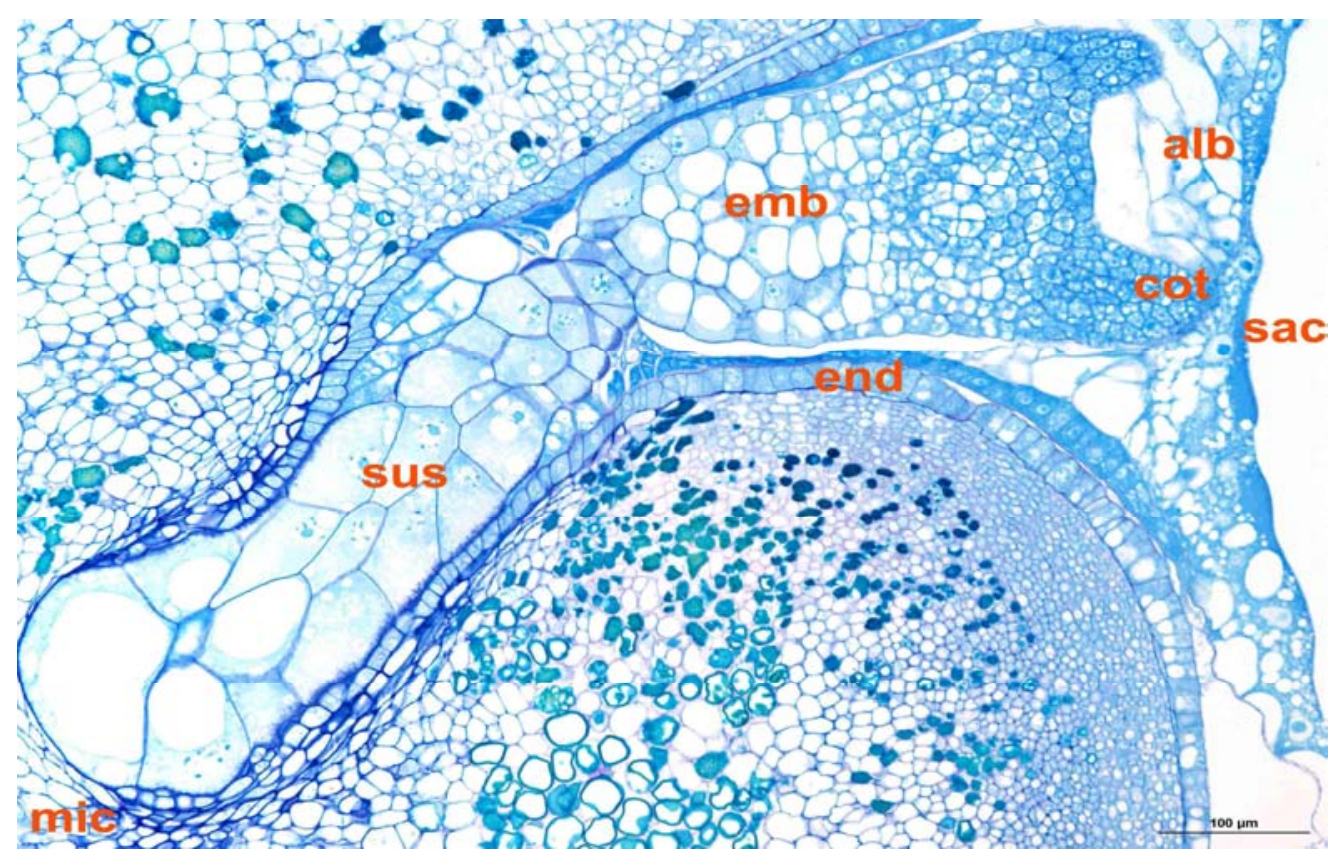

Figure 9: Coupe longitudinale médiane dans l'ovule de P. coccineus montrant un embryon (emb) cotylédonaire jeune. Les cellules basales du suspenseur (sus) sont enflées du côté du micropyle (mic). De l'albumen (alb) cellulaire est encore visible dans le sac embryonnaire (sac). Les cotylédons (cot) commencent à croître. L'épaisseur de l'endothélium (end) est irrégulière. 
maturation de l'embryon et de la dessiccation de la graine qui précèdent la dormance.

La division initiale et transversale du zygote aboutit à la formation de deux entités : une cellule basale de grande taille destinée à devenir le suspenseur et une cellule apicale moins volumineuse qui sera le futur embryon. Comme chez la plupart des autres végétaux, le suspenseur jouera un rôle important dans l'alimentation de l'embryon grâce à sa fonction de synthèse hormonale et au transit des éléments nutritifs du tissu maternel vers l'embryon proprement dit (Yeung et Meinke, 1993). Il est par excellence le siège d'approvisionnement de l'embryon durant la phase précoce de l'embryogenèse. Dès l'apparition des cotylédons, ceux-ci deviennent le site le plus important de transit de nutriments, jusqu'à la maturation de l'embryon. Le rôle de transport du suspenseur s'arrêterait au stade cordiforme tardif (Yeung et Meinke, 1993).

Après la double fécondation, le proembryon s'entoure d'albumen coenocytique (Figure 5). Un jour plus tard, le nombre de cellules du proembryon a augmenté (Tableau 1). Les cellules du suspenseur et de l'embryon proprement dit se différencient singulièrement. Des vaisseaux secondaires latéraux apparaissent dans le tégument externe, de part et d'autre du plan médian de l'ovule. Des structures procambiales s'étendent aussi le long du sac embryonnaire, à partir de la région chalazienne, jusqu'à la région micropylaire. La différenciation et la maturation du tissu vasculaire continuent durant les jours suivant la pollinisation, dans le tégument externe. L'albumen se trouvant au voisinage de l'embryon globulaire se cellularise. Ce phénomène s'intensifie rapidement.

Le tissu nucellaire dégénère progressivement, au fur et à mesure que l'embryon grandit dans le sac embryonnaire (Figure 6). Puis, les cellules des couches moyennes du tégument externe et les couches internes se joignant au tégument interne se relâchent graduellement.

Les divisions des cellules du suspenseur ralentissent par la suite (Tableau 1). Après, le tissu nucellaire est uniquement visible dans la région chalazienne. Des filaments continus des vaisseaux apparaissent dans les parties latérales de l'ovule, et la vascularisation s'intensifie du côté chalazien.

L'initiation des cotylédons débute cinq jours après la pollinisation dans certains cas (Figure 8). À ce moment, le sac embryonnaire est rempli progressivement par la croissance de l'embryon (Figure 9). On remarque des divisions anticlinales des cellules qui se vident graduellement d'amidon.

Au-delà des stades morphologiques de développement des embryons, les deux principales structures embryonnaires (suspenseur et embryon proprement dit) changent progressivement de dimensions. L'évolution de la taille de ces structures

Tableau 1: Evolution de la taille moyenne des principaux paramètres de l'embryon et du suspenseur entre 3 et 12 JAP chez le cultivar X484, de P. vulgaris.

\begin{tabular}{|c|c|c|c|c|c|}
\hline \multirow{2}{*}{$\begin{array}{l}\text { Nombre de jours } \\
\text { après } \\
\text { pollinisation } \\
\text { (JAP) }\end{array}$} & \multicolumn{5}{|c|}{ Paramètres mesurés (Données personnelles) } \\
\hline & $\begin{array}{l}\text { Nombre de } \\
\text { cellules du } \\
\text { suspenseur }\end{array}$ & $\begin{array}{l}\text { Surface du } \\
\text { suspenseur } \\
\left(\mu \mathbf{m}^{2}\right)\end{array}$ & $\begin{array}{c}\text { Longueur du } \\
\text { suspenseur } \\
(\mu \mathrm{m})\end{array}$ & $\begin{array}{c}\text { Longueur de } \\
\text { l'embryon }(\mu \mathrm{m})\end{array}$ & $\begin{array}{c}\text { Largeur de } \\
\text { l'embryon }(\mu \mathrm{m})\end{array}$ \\
\hline 3 & $3 \pm 1$ & $2982 \pm 345$ & $78 \pm 9$ & $151 \pm 16$ & $51 \pm 4$ \\
\hline 4 & $4 \pm 1$ & $3938 \pm 538$ & $93 \pm 4$ & $204 \pm 17$ & $61 \pm 7$ \\
\hline 5 & $6 \pm 1$ & $9402 \pm 1296$ & $100 \pm 18$ & $257 \pm 18$ & $84 \pm 4$ \\
\hline 6 & $12 \pm 2$ & $15573 \pm 1706$ & $154 \pm 43$ & $459 \pm 67$ & $97 \pm 10$ \\
\hline 7 & $14 \pm 2$ & $17748 \pm 1391$ & $272 \pm 26$ & $599 \pm 48$ & $127 \pm 28$ \\
\hline 8 & $16 \pm 2$ & $25167 \pm 1652$ & $361 \pm 54$ & $878 \pm 155$ & $393 \pm 25$ \\
\hline 9 & $26 \pm 3$ & $36931 \pm 3915$ & $380 \pm 57$ & $933 \pm 98$ & $387 \pm 37$ \\
\hline 10 & $13 \pm 2$ & $32353 \pm 3122$ & $353 \pm 36$ & $1179 \pm 147$ & $625 \pm 97$ \\
\hline 11 & $16 \pm 1$ & $24991 \pm 2393$ & $311 \pm 47$ & $2276 \pm 558$ & $792 \pm 142$ \\
\hline 12 & $14 \pm 1$ & $26270 \pm 2986$ & $709 \pm 83$ & $2798 \pm 324$ & $1227 \pm 185$ \\
\hline
\end{tabular}


entre 3 et 12 jours après pollinisation (JAP) est donnée dans le tableau 1 pour le cultivar X484 de P. vulgaris.

$\mathrm{Au}$ cours du développement de l'embryon, la forme et la longueur de l'embryon sont corrélées. Le stade atteint par l'embryon peut se référer à la fois à ces paramètres et au nombre de jours après la pollinisation. Ces observations concordent avec les travaux de Yeung et al. (2001).

\section{Embryogenèse de $P$. coccineus}

Les Figures 7,8 et 9 illustrent le développement embryonnaire chez l'espèce $P$. coccineus. Les embryons présentent une base suspensoriale hypertrophiée par rapport à l'embryon proprement dit. La présence du suspenseur stimule la croissance de l'embryon et facilite la régénération de plantules lorsque l'embryon est cultivé in vitro, surtout au stade cordiforme. Le suspenseur est énormément sollicité à ce stade de développement de l'embryon. Cependant, il peut être suppléé par l'usage des régulateurs de croissance (Brady et Combs, 1998 ; Ciavatta et al., 2001; Weijers et Jürgens, 2005). Le suspenseur a par contre un effet réduit lorsque l'embryon a atteint le stade cotylédonaire.

Durant l'embryogenèse précoce, le développement du suspenseur est rapide en taille et en poids. À partir du stade préglobulaire (Figure 7), une différenciation structurale est visible avec la formation de parois. Les premières parois apparaissent tout autour du suspenseur au niveau des cellules adjacentes au tapetum tégumentaire, puis elles se propagent vers les cellules internes du suspenseur. Le noyau de la cellule basale du suspenseur est caractérisé par sa grande taille. $\mathrm{Au}$ point de vue histologique, les cellules suspensoriales semblent actives, surtout avant que l'embryon ne dépasse le stade cordiforme (Yeung et al., 2001).

Les différences dans le développement embryonnaire entre $P$. vulgaris et $P$. coccineus se rapportent essentiellement au délai requis pour atteindre des stades morphologiques de développement chez l'embryon puis aux formes et dimensions des suspenseurs. Lors des croisements entre $P$. vulgaris et P.coccineus, les embryons hybrides se développent plus lentement que ceux de P. vulgaris (Nguema et al., 2007). Des différences sont aussi notées dans la forme et les dimensions des suspenseurs entre les génotypes utilisés au sein d'une même espèce.

Les embryons de $P$. coccineus sont plus faciles à observer au microscope car ils sont de plus grande taille que ceux de $P$. vulgaris (Yeung et Meinke, 1993). Cette particularité permet de mettre en évidence le rôle de stimulateur de croissance du suspenseur de ces embryons, avant le stade de développement cordiforme tardif. Au stade proembryonnaire, la différence de coloration, noire en général, entre les cellules suspensoriales et les cellules de l'embryon proprement dit traduit l'intense activité du suspenseur. Dans les stades ultérieurs de développement de l'embryon, il y a un amoindrissement de cette nuance, expliquant de ce fait le ralentissement de l'activité du suspenseur (Yeung et Meinke, 1993; Yeung et al., 2001; Nomizu et al., 2004).

De manière générale, les zygotes avec un suspenseur anormalement développé ont à l'inverse un albumen de petite taille, comme l'ont remarqué Perata et al. (1990). Une attention particulière doit être portée sur le système embryon-suspenseur pour une meilleure connaissance du rôle physiologique du suspenseur.

Chez Phaseolus L., tout comme chez la plupart des angiospermes, la fleur produit deux types de spores dont l'une se développe en grain de pollen et l'autre en sac embryonnaire.

Dès que les gamètes sont matures et que les conditions favorables à la pollinisation sont réunies, les tubes polliniques sont attirés vers l'ovule par une substance protéinique exsudée du micropyle. Cet exsudat provient des synergides et contient aussi du calcium libre, quoique ce dernier ne soit pas impliqué dans l'attraction (Willemse et al., 1996). Le calcium interviendra lors de la fusion des gamètes pour induire l'apparition de la paroi cellulosique après la fécondation. Cette paroi participe à l'établissement de la polarité comme c'est le cas chez l'algue Fucus (Tamborindeguy, 2004) ou le maïs (Antoine et al., 2000).

La pollinisation et la fécondation sont des événements séparés. La pollinisation correspond strictement au transfert du pollen de l'anthère au stigmate, suivi de son parcours dans le tissu stylaire. Elle a lieu à l'intérieur du bouton floral, le jour de l'anthèse. L'étape 
ultime de la pollinisation correspond à la pénétration du tube pollinique à travers le micropyle. Elle est suivie par la libération des noyaux spermatiques à l'intérieur de l'ovule. La fécondation est la fusion des noyaux des deux parents.

Les études moléculaires et génétiques sur les éléments impliqués dans la double fécondation font état d'un développement autonome de l'albumen. Cependant, on note un effet prédominant de gènes gamétophytiques maternels réduisant au silence les gènes paternels lors de la double fécondation selon Raghavan (2003).

Le zygote constitue le point de départ quasi systématique du développement des organismes pluricellulaires. Il subit des divisions mitotiques pour devenir l'embryon tandis que l'albumen cellulaire poursuit parallèlement son développement (Lopes et Larkins, 1993).

De manière générale, le développement embryonnaire chez les angiospermes suit plusieurs étapes. A la suite des divisions cellulaires, le zygote traverse des stades au cours desquels l'embryon présente des formes caractéristiques auxquelles on donne des noms descriptifs pour leur dénomination. Le développement embryonnaire est avant tout une multiplication cellulaire correspondant à l'auxèse, à la mérèse et à l'acquisition de propriétés nouvelles désignée sous le terme de différenciation.

L'embryon est d'abord pré-globulaire, puis globulaire. Cette étape se caractérise par un suspenseur fait d'une grande cellule basale vers le micropyle. L'embryon proprement dit présente une forme globoïde. Une couche de protoderme est visible le long de sa structure. La région distale au micropyle contient l'albumen coenocytique dont le noyau se divise pour produire une masse de cellules albuminées au voisinage de l'embryon.

L'étape de torpille est caractérisée par la courbure des cotylédons pour remplir l'ovule. Lors de cette étape, les trois tissus des méristèmes fondamentaux sont clairement discernables. L'axe central de l'embryon est appelé l'axe hypocotyle-racine. La différenciation se poursuivra dans trois parties: l'épicotyle, l'hypocotyle et la radicule.

Le suspenseur fixe l'embryon et lui transfère des éléments nutritifs au travers des tissus maternels. Il est métaboliquement actif. Il fournit des éléments nutritifs et des régulateurs de croissance (gibbérellines) à l'embryon (Nickle et Meinke, 1998 ; Nguema et al., 2007). En général, il meurt par apoptose pendant l'étape de torpille et il n'est plus présent dans les graines mûres chez certaines espèces végétales. Il est possible que l'embryon commande la substance inhibitrice $\mathrm{du}$ suspenseur et prévienne les cellules embryonnaires de se développer à ses dépens.

L'organisation de l'embryon peut être considérée comme modulaire (Jürgens et al., 1991; Mayer et al., 1993), avec deux programmes génétiques différents. L'un concerne directement les mécanismes d'édification de l'embryon et l'autre initialise le programme génétique de la plante adulte dont il commandera le phénotype (Yazawa et al., 2004). L'action des gènes interfère avec la différentiation tissulaire, indépendamment de la morphogenèse (Losick et Shapiro, 1993).

\section{Conclusion}

L'embryogenèse de $P$. vulgaris est similaire à celle de $P$. coccineus dans son déroulement général.

La connaissance du développement embryonnaire chez les espèces du genre Phaseolus est importante dans l'optique de mieux comprendre et prévoir les mécanismes régissant le développement des embryons hybrides. Ces acquis sont d'autant plus intéressants qu'ils peuvent favoriser l'intervention spécifique pour le sauvetage des embryons hybrides car les barrières d'incompatibilité sont essentiellement postzygotiques au sein du genre Phaseolus.

Les analyses histologiques réalisées sur des embryons aux premiers stades de leur développement sont particulièrement difficiles. En conséquence, le nombre de cellules des embryons et leurs dimensions lors de l'acquisition de la polarité embryonnaire restent à déterminer. Ces résultats justifient la nécessité d'approfondir l'étude histologique de l'embryogenèse chez Phaseolus.

\section{REMERCIEMENTS}

Nous remercions la Faculté universitaire des Sciences agronomiques de Gembloux qui nous a permis de faire ce travail grâce à ses centres de documentation et d'information. 


\section{REFERENCES BIBLIOGRAPHIQUES}

Anderson GJ, Hill JD. 2002. Many to flower, few to fruit: the reproductive biology of Hamamelis virginiana Hamamelidaceae. Am. J. Bot., 89(1): 67-78.

Antoine AF, Faure JE, Cordeiro S, Dumas C, Rougier M, Feijo JA. 2000. A calcium influx is triggered and propagates in the zygote as a wavefront during in vitro fertilization of flowering plants. P. N. A. S., 97: 10643-10648.

Berg RY. 2003. Development of ovule, embryo sac, and endosperm in Triteleia Themidaceae relative to taxonomy. Am. $J$. Bot., 90(6): 937-948.

Brady T, Combs SH. 1998. The suspensor is a major route of nutrients into proembryo, globular and heart stage Phaseolus vulgaris embryos. Biology Department, Hamilton College Clinton, U.S.A, 419424.

Ciavatta VT, Morillon R, Pullman GS, Chrispeels MJ, Cairney J. 2001. An aquaglyceroporin is abundantly expressed early in the development of the suspensor and the embryo proper of Loblolly Pine. Plant Physiology, 127: 1556-1567.

Derksen J, Kuiman B, Hoedemaekers K, Guyon A, Bonhomme S, Pierson ES. 2002. Growth and cellular organization of Arabidopsis pollen tubes in vitro. Sex. Plant Reprod., 15: 133-139.

Farjon A, Ortiz GS 2003. Cone and ovule development in Cunninghamia and Taiwania Cupressaceae sensu lato and its significance for conifer evolution. Am. J. Bot., 90(1): 8-16.

Gallois P. 2001. Future of early embryogenesis studies in Arabidopsis thaliana. C.R. Acad. Sci. Paris, Sciences de la Vie, 324: 569-573.

Grini PE, Jürgens G, Hülskamp M. 2002. Embryo and endosperm development is disrupted in the female gametophytic capulet mutants of Arabidopsis. Genetics, 162: 1911-1925.

Jürgens G, Mayer U, Torres Ruiz R, Berleth T, Misera S. 1991. Genetic analysis of pattern formation in the Arabidopsis embryo. Dev. Suppl., 1: 27-38.

Ledesma N, Sugiyama N. 2005. Pollen quality and performance in strawberry plants exposed to high-temperature stress.
Journ. Amer. Soc. Hort. Sci., 130(3): 341347.

Lopes A, Larkins BA. 1993. Endosperm origin, development, and function. The Plant Cell, 5: 1383-1399.

Losick R, Shapiro L.1993. Checkpoints that couple gene expression to morphogenesis. Science, 262: 1227-1228.

Massardo F, Corcuera L, Alberdi M. 2000. Seed physiology, production and technology: Embryo Physiological Responses to Cold by Two Cultivars of Oat during Germination. Crop Science, 40: 1694-1701.

Mayer U, Büttner G, Jürgens G. 1993. Apicalbasal pattern formation in the Arabidopsis embryo: studies on the role of the gnom gene. Development, 117: 149-162.

Nguema Ndoutoumou P, Toussaint A, Baudoin JP. 2009. Development of HY1 hybrid embryos between a cultivar of Phaseolus vulgaris L. and a wild genotype of Phaseolus coccineus L. Int. J. Biol. Chem. Sci., 3(1): 20-29.

Nguema Ndoutoumou P, Toussaint A, Baudoin JP. 2007. Embryogenèse comparative lors des croisements entre Phaseolus coccineus L. et Phaseolus vulgaris L. BASE, 11(2): 97-107.

Nickle TC, Meinke DW. 1998. A cytokinesisdefective mutant of Arabidopsis cyt 1 characterized by embryonic lethality, incomplete cell walls, and excessive callose accumulation. Plant Journal, 115: 321-332.

Nomizu T, Niimi Y, Watanabe E. 2004. Embryo development and seed germination of Hepatica nobilis Schreber var. japonica as affected by temperature after sowing. Scientia Horticulturae, 99: 345-352.

Perata P, Picciarelli P, Alpi A. 1990. Pattern of variations in abscisic acid content in suspensors, embryos, and integuments of developing Phaseolus coccineus seeds. Plant Physiol., 94: 1776-1780.

Pullman GS, Buchanan M. 2003. Loblolly pine Pinus taeda L.: stage-specific elemental analyses of zygotic embryo and female gametophyte tissue. Plant Science, 164: 943-954.

Raghavan V. 2003. Some reflections on double fertilization, from its discovery to 
the present. New Phytologist, 159(3): 565-585.

Sallandrouze A, Faurobert M, El Maâtaouia M. 2002. Characterization of the developmental stages of cypress zygotic embryos by two-dimensional electrophoresis and by cytochemistry. Physiol. Plant, 114: 608-618.

Shi Z, Stösser R. 2005. Reproductive biology of Chinese chestnut Castanea mollissima Blume. Europ. J. Hort. Sci., 70(2): 96103.

Soltis PS, Soltis DE. 2004. The origin and diversification of angiosperms. Am. J. Bot., 91(10): 1614-1626.

Sornsathapornkul P, Owens JN. 1999. Zygotic embryo development in a tropical Acacia hybrid Acacia mangium Willd $\mathrm{x} A$. auriculiformis A. Cunn. Ex Benth. Int. J. Plant Sci., 160(3): 445-458.

Stauffer FW, Rutishauser R, Endress PK. 2002. Morphology and development of the female flowers in Geonoma interrupta Arecaceae. Am. J. Bot., 89(2): 220-229.

Tamborindeguy C. 2004. Analyse par les approches de la génomique fonctionnelle de l'induction de la polarité embryonnaire chez le tournesol. Ecole Nationale Supérieure Agronomique de Toulouse France, 285p.

Teixeira SP, Carmello-Guerreiro SM, Machado SR. 2004. Fruit and seed ontogeny related to the seed behaviour of two tropical species of Caesalpinia Leguminosae. Botanical Journal of the Linnean Society, 146: 57-70.

Weijers D, Jürgens G 2005. Auxin and embryo axis formation: the ends in sight? Current Opinion in Plant Biology, 8: 3237.

Whittier DP, Braggins JE. 2000. Observations on the mature gametophyte of Phylloglossum Lycopodiaceae. Am. J. Bot., 87(7): 920-924.

Willemse MTM, Wittich PE, Owens SJ, Rudall PJ. 1996. Pollination and fertilisation in Gasteria verrucosa: interaction between pollen tube and ovary. Reproductive biology in systematics, conservation and economic botany. Proceedings of a conference, Kew, Richmond, UK, 2-5 September 1996, 57-69.

Yazawa K, Takahata K, Kamada H. 2004. Isolation of the gene encoding Carrot leafy cotyledon 1 and expression analysis during somatic and zygotic embryogenesis. Plant Physiol. Biochem., 42: 215-223.

Yeung EC, Meinke DW. 1993. Embryogenesis in Angiosperms: Development of the suspensor. The Plant Cell, 5: 1371-1381.

Yeung EC, Meinke DM, Nickle TC. 2001. Embryology of flowering plants - an overview. Phytomorphology, Golden Jubilee Issue: 289-304. 